Chronic Obstructive Pulmonary Diseases: Journal of the COPD Foundation

\title{
Editorial
}

\section{Clinical Trial Design for Alpha-1 Antitrypsin Deficiency: A Model for Rare Diseases}

\author{
Michael R. Jacobs, Pharm D ${ }^{1}$ Gerard J. Criner, MD $^{1}$
}

Abbreviations: alpha-1 antitrypsin deficiency, AATD; Code of Federal Regulations, CFR; Food and Drug Administration, FDA; National Organization for Rare Disorders, NORD;

Citation: Jacobs MR, Criner GJ. Editorial- Clinical trial design for alpha-1 antitrypsin deficiency: a model for rare diseases. J COPD F. 2015;2(2): 91-93. doi: http://dx.doi.org/jcopdf.2.2.2015.0140

1 Department of Thoracic Medicine and Surgery, Temple University School of Medicine, Philadelphia, Pennsylvania

\section{Address correspondence to:}

Michael R. Jacobs, PharmD

Temple University School of Pharmacy

Director of Clinical Research

Temple Lung Center

Phone: (215)707-2242

Email: Michael.jacobs@tuhs.temple.edu

\section{Keywords:}

alpha-1 antitrypsin deficiency; clinical trial design; rare diseases: adaptive clinical trials; surrogate endpoints

In this issue of the Journal, Wanner and others summarize the results of a 2-day conference that addressed the challenges and possible approaches to conducting meaningful research on a rare disease, alpha- 1 antitrypsin deficiency (AATD). There are about 7000 rare diseases and the same obstacles must be faced in researching any of these conditions. The ideas that emanated from this conference in conducting AATD research are not, in and of themselves, new. What is novel is that, for the first time, alpha stakeholders have come together to begin a process that could optimize the way alpha-1 antitrypsin deficiency research is performed. The conclusions identified at the conference are similar to those recommended for research on other rare diseases: 1) use of flexible or adaptive clinical trial methodologies; 2) performing natural history studies and identifying biomarkers as surrogates of disease progression; and 3) generating and sharing information through patient registries and databases. Each of the suggested approaches has the potential to accelerate development of treatments for this genetic condition. Unfortunately, each comes with its own set of potential problems and limitations.

The Code of Federal Regulations (CFR) requires "adequate and well-controlled studies" for the approval of drugs. In general, this has been interpreted to mean prospective, randomized, blinded, controlled trials. The Director of the Center for Drug Evaluation and Research under this section of the CFR is given the authority to waive some or all of the requirements for adequate and well-controlled studies. The Food and Drug Administration (FDA) has shown this flexibility and has been recognized by the National Organization for Rare Disorders (NORD) for providing alternative approval pathways for rare disease drugs since the introduction of the Orphan Drug Act. ${ }^{1}$ It should be noted that in this NORD report that about one-third of drug approvals for rare disease were received based on the accepted criterion of 2 positive randomized controlled trials. In other cases a single randomized trial was accepted, historical controls were used, or the sponsor supported an application based on existing medical literature. While the FDA has demonstrated its ability to be flexible in the types of evidence needed for drug approval, a randomized controlled trial remains the gold standard as shown in the Zemaira ${ }^{\circledR}$ example provided by Wanner, et al as well as in the recent approvals of nintedanib and pirfenidone for the treatment of idiopathic pulmonary fibrosis, another rare disease. 
Adaptive clinical trial designs may have a role in rare disease research. However, it is important to understand that the primary motivations for using adaptive designs are to save time and improve resource utilization. This is why adaptive clinical trials are of particular interest to the pharmaceutical industry where accelerating drug development and controlling costs are major concerns. Slow enrollment into a trial can negate these potential benefits to the sponsor. While fewer patients may be needed in an adaptive trial design, realizing this objective is often only known once the trial is completed and is not the primary goal. As the authors point out, the likelihood of successfully executing an adaptive clinical trial requires careful planning and can be estimated through the use of computer simulations. This approach makes sense in the pharmaceutical industry where saving a year of drug development time can translate into millions of dollars. Because of their complexity, the decision to use an adaptive clinical trial design must be carefully considered.

The second approach suggested at the conference to facilitate research in AATD was to identify biomarkers that correlate with disease progression that could be used as surrogate endpoints. As pointed out by the authors, some biomarkers may not be specific to AATD and others may emerge only later in the trajectory of the disease (such as the functional biomarkers) and may not be of substantial value beyond current outcome measures. Biochemical and molecular biomarkers have the potential to be important indicators of the earliest effects of an intervention; however these, too, may not be specific to AATD. Furthermore, much additional research needs to be done to show that changes in the level or activity of these potential biomarkers correlates with a change in the progression of disease. This information can best be obtained through the welldesigned natural history studies also called for at this conference.

Possibly the most significant recommendation to come out of this conference, and the one most likely to have an immediate impact, was the call for increased integration and collaboration among AATD researchers. The elements of this collaboration should include standardized data definitions and reporting requirements, increased availability of patient registries and databases, recommended clinical trial endpoints, and what might be called an "adaptive clinical research program." Alpha- 1 antitrypsin deficiency research needs to mimic the approach used by the pharmaceutical industry. While hundreds of drug candidates may be identified by a company, only those that show the potential for success are moved forward and receive additional funding. It also means that once a drug is ready to be evaluated in a clinical trial that multiple investigators at multiple sites agree to follow the same protocol and measure the same outcomes. This approach should work equally well to identify potential biomarkers. This doesn't mean that funding for other chemical entities or biomarkers is abandoned. It does mean that through collaboration, patients with AATD have the best chance of receiving effective treatment options.

The research approaches discussed at the AATD conference could become increasingly relevant to COPD research in general. Performing clinical trials in COPD is challenging because the disorder is hallmarked by complexly ill patients with protean manifestations that has no biomarker that can be used as a surrogate marker for outcomes. Hence, performing clinical trials in COPD is inefficient and challenging because it requires substantial numbers of patients followed for prolonged periods to effectively evaluate responses to treatment. As with AATD, it is likely that COPD clinical phenotypes, and eventually endotypes, will be identified that specifically target unique pathophysiologic processes and provide targeted treatment options and facilitate novel therapeutic development. The American Thoracic Society and the European Respriatory Society recently released a statement on research questions in $\mathrm{COPD}^{2}$ which includes recommendations for studying COPD phenotypes in the context of treatment responses and clinical outcomes. This sensible methodology should result in more broadly applicable treatment approaches. 


\section{References}

1. Sasinowski FJ. Quantum of effectiveness, evidence in FDA's approval of orphan drugs. National Organization of Rare Diseases website.

https://www.rarediseases.org/docs/policy/NORDstudyofFDA approvaloforphandrugs.pdf Accessed April 2015.

2. Celli BR, Decramer M, Wedzicha JA, et al for the ATS/ERS Task Force for COPD Research. An official American Thoracic Society/ European Respiratory Society Statement: Research questions in chronic obstructive pulmonary disease. Am J Respir Crit Care Med. 2015; 191(7): e4-e27. doi: http://dx.doi.org/10.1164/rccm.201501-0044ST 